\title{
Postnatal care of the woman and her baby during hospital stay; bridging the gap in reproductive healthcare
}

\author{
Dasanayake DLW, Goonewardene IMR \\ Department of Obstetrics \& Gynaecology, Faculty of Medicine, University of Ruhuna, Galle, Sri Lanka. \\ Correspondence: Dr. Lanka Dasanayake \\ e-mail: lanka.dasanayake@yahoo.com \\ https://orcid.org/0000-0001-9306-6508 \\ Submitted on 02.11.2020 and accepted for publication on 19.11.2020
}

\begin{abstract}
Postnatal care of the woman and her newborn baby, is the weakest and the most neglected component of reproductive health care. As the first day of the postpartum period carries the highest risk of adverse outcomes for the woman and her baby, it is essential that comprehensive postnatal care is initiated immediately after delivery and continued until the woman is discharged from hospital. The aspects of postnatal care which should be initiated on the first postpartum day itself include: pain management; perineal care; bladder care; care of the new born; postpartum contraception; lactation management; and assessment of psychological wellbeing. The World Health Organisation (WHO) has issued detailed, evidence-based recommendations for postnatal care. These WHO recommendations, as well as other, evidence-based recommendations, especially for postpartum contraception, are feasible and applicable in Sri Lanka, and therefore should be implemented.
\end{abstract}

Keywords: Postnatal care, postpartum contraception, postpartum intra uterine device

\section{Introduction}

Reproductive health care (RHC) includes antenatal, intrapartum, postnatal and neonatal care, and provision of family planning services. Of these five components of RHC, postnatal care is the weakest and the most neglected component, and it has been found that women are least likely to be satisfied with postnatal care, compared to the other components. Global data show that almost half the postnatal maternal deaths occur within the first 24 hours of child birth, about two thirds of maternal deaths occur during the first week, and approximately one million newborns die on the $1^{\text {st }}$ day of their birth (1). Therefore, as the first 24 hours after birth is a critical phase in the life of the mother and her newborn, for the first 24 hours after childbirth (the immediate postnatal period) the mother and baby should remain in the hospital as this would provide an opportunity to safeguard the health and survival of the mother and her baby (1).

Optimising the health of women and their newborns should commence in the immediate postnatal period, not as a single encounter with the healthcare team, but as an ongoing process, tailored to each woman's individual needs. Although globally, only about $75 \%$ of women have a skilled birth attendant during childbirth, in Sri Lanka, more than $99 \%$ of births are attended on by skilled personnel in a hospital (2). Therefore, Sri Lanka has an excellent opportunity to extend intrapartum care to comprehensive postnatal care, until the woman and her baby are discharged from hospital.

The objective of this review is to identify and recommend evidence-based best practices which 
should be implemented soon after delivery of the baby and from then on until the mother and baby are discharged from the hospital.

A comprehensive literature search was carried out to identify and extract information from relevant publications in the Cochrane Database, PubMed and Google Scholar up to $30^{\text {th }}$ October 2020. The search terms used were: "postnatal AND care", "postpartum AND care", postpartum AND immediate", "postpartum AND intra-uterine device", "postpartum AND contraception". From the identified publications relevant citations were also identified and further information extracted.

\section{Recommendations}

During the transition from intrapartum care to postpartum care in the hospital, several components of care should be addressed. These include: immediate postpartum monitoring; management of delivery-related pain; perineal care; bladder care; care of the new born; postpartum contraception; lactation management; assessment of psychological wellbeing; and health education and counselling. All postnatal women and their new born babies should stay at a healthcare facility for at least $24 \mathrm{hrs}$ after delivery. During this period these women should have assessment of vaginal bleeding, retraction of uterus, fundal height, temperature, heart rate, blood pressure and voiding of urine, starting from immediately after birth, at appropriate intervals, depending on the clinical situation.

\section{Immediate postpartum monitoring}

The use of the Modified Obstetric Early Warning System (MOEWS), which alerts care providers of potential impending critical illnesses, is recommended to improve maternal outcomes and safety (3). The MOEWS chart, which is found on the reverse side of the national partogram of Sri Lanka, should be used to monitor the mother for two hours after vaginal delivery and four hours after caesarean delivery. As abnormalities in physiological parameters precede critical illnesses, the 'track and trigger' method used in this chart can aid in recognition of maternal morbidity at an early stage, thereby preventing escalation of the cascade into severe maternal morbidity and mortality.

\section{Discomfort and pain}

Almost all mothers experience perineal pain in varying severity following child birth, and the provision of safe and effective pain relief is an essential component of postnatal care. However, this is one of the most ignored aspects following childbirth, due to the under estimation of the mother's pain and discomfort, which could be severe enough to interfere with mobilisation and bonding with the new born. It could also lead to acute urinary retention, constipation and disturbances in breastfeeding. The perineal pain and discomfort often lasts for 10 to 12 days, is more severe in the immediate postnatal period, and in some will last for several months following delivery.

Acetaminophen and non-steroidal antiinflammatory drugs (NSAIDs) such as ibuprofen are relatively safe during breastfeeding, and they act effectively on both perineal discomfort and uterine cramping. Many other analgesics are secreted in breast milk and therefore should be avoided. Rectal NSAIDs could be routinely offered to women following childbirth, especially after perineal repair of first- and second-degree trauma provided these drugs are not contraindicated (4). Immediately after delivery, ice packs may help reduce pain and oedema at the site of an episiotomy or repaired laceration. A local anaesthetic e.g. lidocaine cream or spray can also be used to relieve pain. After surgery or repair of significant lacerations, women may require opioids to relieve their pain. If pain is significantly worsening, women should be evaluated for complications such as vulval haematoma.

\section{Wound care}

It has been estimated that, globally more than $85 \%$ women will sustain some degree of perineal trauma (spontaneously or due to an episiotomy) and that $60-70 \%$ of them will require suturing (5). In Sri Lanka, almost all primipara and many multipara have an episiotomy. Laxatives should be 
administered, in the immediate postpartum period, if a woman has painful defaecation or constipation following perineal trauma, to aid easier bowel motions and early discharge from hospital. Broad spectrum, prophylactic antibiotics are recommended for third- or fourth-degree perineal tear but not for first- and second-degree perineal tears or episiotomies (1). Women should be encouraged to have a shower (preferable) or bath (including a sitz bath if they wish to). However, vaginal douching is not recommended in the early puerperium. The vulva should be cleaned from anterior to posterior and not vice versa, to prevent the possibility of faecal contamination of traumatised areas. Caesarean wound care includes: removing the dressing 24 hours after the caesarean delivery; assessing the wound for signs of infection (such as increasing pain, redness or discharge), separation or dehiscence; re-application of soft dressing, encouraging the woman to wear loose, comfortable clothes and cotton underwear; gently cleaning and drying the wound daily and if needed; planning the removal of sutures or clips (6).

\section{Bladder and bowel care}

The bladder could be an unfortunate victim of childbirth. Varying degrees of bladder insults are possible as a result of denervation and ischaemia of bladder muscles during vaginal delivery. The incidence of voiding dysfunction is reported to range from $0.7 \%$ to $4 \%$ of all deliveries, while if uroflowmetry and post micturition ultrasonography are carried out during the immediate postnatal period, abnormal voiding parameters may be identified in up to $43 \%$ of postnatal women (7). If the abnormality goes unrecognized, there is a possibility of it leading to recurrent urinary tract infections and urinary stress incontinence. It is recommended that, no women should be allowed to go longer than six hours without voiding (1). Postnatal women with following risk factors are more vulnerable for voiding dysfunction: primipara; instrumental vaginal delivery; epidural anaesthesia; prolonged latent phase; and perineal trauma. Early recognition of urinary retention is important to prevent long term complications of bladder dysfunction. Simple instructions should be given to every postnatal woman to empty the bladder every $4-6$ hours and to inform the maternity team if any voiding difficulty is encountered. If over distension occurs, catheterisation is necessary to promptly relieve discomfort and to prevent long-term urinary dysfunction. If over distension recurs, an indwelling or intermittent catheter may be needed.

Women should be encouraged to defaecate before leaving the hospital, although with early discharge, this recommendation is often not possible. If defecation has not occurred within two to three days, a mild purgative (e.g. bisacodyl suppositories) can be given. Avoiding constipation can prevent or help relieve existing haemorrhoids, which can also be treated with warm sitz baths. Women with an extensive perineal laceration repair involving the rectum or anal sphincter can be given stool softeners (e.g. docusate). Neuraxial (spinal or epidural) or general anaesthesia may delay defaecation and spontaneous urination, in part by delaying ambulation.

\section{Care of the newborn}

Clean, dry cord care is recommended. A complete clinical examination of the baby, around one hour after birth, when the baby has had his / her first breastfeed, and again before discharge, is needed. Bathing of neonate should be delayed until 24 hours after birth, and if this is not possible due to cultural reasons, bathing should be delayed for at least six hours. The clothing of the baby should be appropriate for the ambient temperature. The mother and baby should stay in the same room 24 hours a day, unless the baby needs special care. The mother should communicate and play with the newborn. Immunisation should be promoted as per existing WHO guidelines. Preterm and low-birth-weight babies should be identified immediately after birth and should be provided special care as per existing WHO guidelines. On discharge, the baby should not have signs of infection or other diseases, and should be breastfeeding well(1). 


\section{Postpartum contraception}

Closely spaced pregnancies result in adverse maternal perinatal and infant out comes. Post Partum Family Planning (PPFP) enables women to achieve healthy intervals between births and it is estimated to potentially prevent $25-40 \%$ of maternal deaths and reduce child mortality by about $10 \%(8,9)$. Fertility returns shortly after childbirth, and ovulation may occur as early as 3 - 4 weeks postdelivery in non-breastfeeding mothers (10). As up to $50 \%$ women may resume sexual activity within the first six weeks after a delivery, the immediate period of postnatal stay in a hospital would provide the best opportunity to provide PPFP, and this could also overcome difficulties that women may face in accessing appropriate contraceptive services later on.

Discussions on PPFP should have been carried out antenatally. Further discussions and the provision of PPFP according to woman's wishes, should be initiated soon after delivery. For women with no other medical complications, there is no restriction for the use of the following methods during the immediate postpartum period: postpartum intrauterine device (PPIUD); progestogen implants; progesterone only pill (11). The PPIUD has been shown to be feasible and safe in different settings (12), and it is recommended that the PPIUD is offered to women before leaving hospital (i.e. within 48 hours) (13).

\section{Lactation management}

Breastfeeding is considered as the single most important low-cost intervention to reduce child morbidity and mortality worldwide (14). While artificial feeding increases the risk of developing common diseases such as diarrhoea and pneumonia, which are leading causes of child mortality, breastfeeding could reduce nearly $50 \%$ of diarrhoea and a third of respiratory tract infection, and long-term health risk such as childhood obesity and diabetes in children. It has been estimated that scaling up breastfeeding to a near universal level could prevent more than 800,000 childhood deaths per annum. Furthermore, "breast feeding is an integral part of the reproductive cycle for women and it has important positive effects on maternal heath such as delayed return of fertility and thereby contributing to birth spacing, as well as a lower risk of breast and ovarian cancers later on in life (15).

Support must be immediately available for new mothers to initiate breastfeeding soon after delivery, in the hospital. Extended support could be offered in several ways by trained personnel: during antenatal or postnatal care; scheduled postnatal visits so that women can predict when support will be available; and support tailored to the setting and the needs of the population group. Support is likely to be more effective in settings with high initiation rates. The most important step for encouraging breastfeeding practice is "Baby Friendly" hospitals. The global Baby Friendly Hospital Initiative which is a complex intervention incorporating 10 steps to successful breastfeeding, has been shown to be associated with increased breastfeeding rates (16). Improving access to skilled breastfeeding counseling and education has been shown to result in up to $90 \%$ increase in exclusive breastfeeding rates for infants up to five months of age. Access to maternity leave has also been found to increase exclusive breastfeeding rates by about $50 \%$, and has even been linked to lower infant mortality in some countries (17).

Sri Lanka ranks first among 97 countries globally on breastfeeding rates according to a survey conducted by the World Breastfeeding Trends Initiative in 2019, achieving first "Green" nation status in supporting breastfeeding women (18). In 2016, initiation of breast feeding within first hour was $90 \%$, and prevalence of exclusive breastfeeding up to six months was $82 \%$, in Sri Lanka (2).

\section{Psychological wellbeing}

Childbirth is a time of great vulnerability for a woman to become mentally unwell. It is a good practice to assess the postpartum women immediately after delivery for early recognition purposes, although the affective disorders usually take more than 24 hours to manifest. Mild transient mood disturbances such as tearfulness, anxiety, irritation and restlessness may occur in up to $70 \%$ of women, commonly during the first week after delivery. Affective disorders range in severity from these early "postpartum blues" to postpartum 
psychosis, a serious state affecting less than $1 \%$ of mothers. Postpartum depression, a condition often exhibiting the disabling symptoms of dysphoria, emotional lability, insomnia, confusion, anxiety, guilt, and suicidal ideation, within this spectrum (19).

Physicians should ask women about symptoms of depression before and after delivery and be alert to recognize symptoms of depression, which may resemble the normal effects of new motherhood (e.g. fatigue, difficulty in concentrating). A preexisting mental disorder, including prior postpartum depression, is likely to recur or worsen during the puerperium. Therefore, affected women should be monitored closely. The Edinburgh Postnatal Depression Scale is by far the most widely used instrument to detect postpartum depression (20). A woman who has lost her baby should receive additional supportive care.

\section{Postnatal Education and Counseling}

Postnatal education and counselling is important, should complement antenatal education and counselling, and should be implemented prior to discharge from the hospital. Ideally, postnatal education and counselling needs to be individualised and flexible, although there could be barriers to do so (1). Women could have anxieties and fears around early parenting and their changing role, and the post natal healthcare providers need to recognise this and ensure care is individualised to address each woman's / family's particular concerns.

\section{Conclusions}

Comprehensive postnatal care for women after childbirth, is a vital component of reproductive health care which is often neglected. The first 24 hours after childbirth, which should be spent in the hospital, irrespective of the mode of delivery, represents a critical window period for the clinicians to prevent, detect early, and manage postpartum complications, as well as to educate, counsel and provide appropriate contraceptive services, before they are discharged home. It is essential to improve and provide the best postnatal care possible, and safeguard the immediate as well as the long-term health of mothers and their newborns.

\section{References}

1. World Health Organisation. WHO Recommendations on Postnatal Care of the Mother and Newborn. 2013. WHO. Geneva, Switzerland.

2. Department of Census and Statistics Sri Lanka. Sri Lanka Demographic and Health Survey 2016. Colombo, Sri Lanka. http://www.statistics.gov.1k/Resource/en/ Health/DemographicAndHealthSurveyReport-2016Contents. (Accessed on 30.09.2020).

3. Singh S, McGlennan A, England A, Simons R. A validation study of the CEMACH recommended modified early obstetric warning system (MEOWS). Anaesthesia. 2012; 67: $12-18$.

4. National Institute for Health and Care Excellence. Intrapartum Care of healthy women and their babies during childbirth NICE Clinical Guideline 190, 2014. https://www.nice.org.uk/guidance/cg190/resources/ intrapartum-care-for-healthy-women-and-babies (Accessed on 30.09.2020)

5. Kettle C, Tohill S. Perineal care. BMJ Clin Evid. 2008; 24: 1401.

6. National Institute for Health and Care Excellence. Surgical site infections: prevention and treatment NICE Guideline NG 125 2019. www.nice.org.uk/guidance/ng 125 (Accessed on 30.09.2020)

7. Ian N Ramsay, Torbet TE. Incidence of abnormal voiding parameters in the immediate postpartum period. Neurology and Urodynamics. 1993; 12: 179-183.

8. Campbell MR, Graham, WJ Strategies for reducing maternal mortality. Getting on with what works. The Lancet. 2006; 368(9543): 1284-1299.

9. Cleland J, et al. Family planning .The unfinished agenda. The Lancet. 2006; 368(9549): 1810- 1827.

10. Jackson E, Glasier A. Return of ovulation and menses in postpartum non lactating women: a systematic review. Obstet Gynecol. 2011; 117(3): 657-62.

11. UK Medical Eligibility Criteria. 2016. (Accessed on 30.09.2020).

12. Grimes D, Schulz K, Van Vliet H, Stanwood N. Immediate post-partum insertion of intrauterine devices. Cochrane Database Syst Rev. 2003; (1): CD003036. doi: 10.1002/ 14651858.CD003036. Update in: Cochrane Database Syst Rev. 2010; (5): Cd003036. 
13. Best practice in post-partum family planning paper. No. 1, Royal College of Obstetricians and Gynaecologists, 2015. https://www.rcog.org.uk/en/guidelines-research-services/ guidelines/bpp1/ (Accessed on 30.09.2020).

14. Bhutta ZA, Das JK, Rizvi A, Gaffey MF, Walker N, Horton $\mathrm{S}$, et al. Maternal and Child Nutrition Study Group. Evidence-based interventions for improvement of maternal and child nutrition: what can be done and at what cost? The Lancet. 2013; 382(9890): 452.

15. Victora CG, Bahl R, Barros AJ, França GV, Horton S, Krasevec $\mathrm{J}$, et al. Breastfeeding in the $21^{\text {st }}$ century: epidemiology, mechanisms, and lifelong effect. The Lancet. 2016; 387(10017): 475-90.

16. World Health Organization. United Nations Children's Fund. The Baby-Friendly Hospital Initiative: A Global Effort to Give Babies the Best Possible Start. 1991. WHO. Geneva, Switzerland.
17. Sinha B, Chowdhury R, Sankar MJ, Martines J, Taneja S, Mazumder S, et al. Interventions to improve breastfeeding outcomes: A systematic review and meta-analysis. Acta Paediatrica. 2015; 104: 114-34.

18. World Breastfeeding Trends Initiative year 2019. https://www.worldbreastfeedingtrends.org/wbti-countryranking.php. (Accessed on 30.098.2020).

19. Stocky A, Lynch. J. Acute psychiatric disturbance in pregnancy and the puerperium. Best Pract Res Clin Obstet Gynaecol. 2000; 14(1): 73-87.

20. Cox JL, Holden JM, Sagovsky R. Detection of postnatal depression: Development of the 10 - item Edinburgh Postnatal Depression Scale. British Journal of Psychiatry. 1987; 150: 782-786.. 